\title{
Integrated Hydrodynamic and Oil Spill Modeling using OILMAP Software for Environment Protection of Oil Spill in Cilacap Regency
}

\author{
Chiquita Tri Rezki ${ }^{1}$, Tri Edhi Budhi Soesilo ${ }^{2}$, Herdis Herdiansyah $^{2}$, and Udi Syahnoedi ${ }^{2}$ \\ ${ }^{1}$ Magister Program of Environmental Science, School of Environmental Science, Universitas Indonesia, Salemba - Indonesia \\ ${ }^{2}$ School of Environmental Science, Universitas Indonesia, Salemba - Indonesia
}

\begin{abstract}
Environmental impact due to oil spill comes from several activities such as oil and gas business unit, port facilities, and shipping line. Cilacap regency as one of the areas with high marine productivity in Indonesia has a history of oil spill incident during 2000-2018. One of the information needed in determining the oil spill response strategy to minimize the environmental impact of the oil spill in Cilacap regency is the prediction of oil spill movement. The purpose of this paper is to predict the oil spill movement in Cilacap regency. A lot of studies had been conducted on the oil spill, but there is still lacking further study that integrating Geographic Information System (GIS), hydrodynamic and oil spill to identify the potential environmental impact of the oil spill and develop the response strategy. Method use of this paper is a quantitative method, integrated hydrodynamic and oil spill using OILMAP software. Data use of this paper is land water data, current data, wind data, and spill information. Results of this paper are in the northwest monsoon, the oil spill moves to east and northeast toward the Indian Ocean. Southeast monsoon, the oil spill moves to west and northwest toward the Segara Anakan Lagoon. This paper will contribute to realizing some oil and gas business unit, port facilities, and shipping industries to concerned about the impact of oil spill and do the sustainable business.
\end{abstract}

Keywords: Oil Spill; OILMAP; Oil Spill Response Strategy; Cilacap Regency.

\section{Introduction}

The oil spill is hydrocarbon/oil released either directly or not directly to sea environment [1]. The oil spill in waters derived from some activity operating in waters as oil and gas business unit, port facilities, and shipping line [2,3]. It is one of the factors that gives the impact of environmental, social, and economic changes [4]. These impact can be minimized with proper oil spill response contingency plan.

Some regions in Indonesia has the potential risk affected oil spill derived from oil and gas business unit, port, and shipping line, one of the regions are Cilacap Regency. These regions are one region has port imports and exports oil industry in the south Java, besides its territorial waters included in the international shipping line. The oil spill incident has happened several time in Cilacap Regency, ie in 2000, 2004, 2007, 2008, 2010, 2015, and 2016.

The oil spill response plan is one of the most important requirements owned by the various business unit which has operations in port and waters [5]. International Maritime Organization (IMO), The International Tanker Owners Pollution Federation Limited (ITOPF), and International Petroleum Industry Environmental Conservation Association (IPECA) organized the guideline of an oil spill contingency plan. Indonesia government also organized some regulations regarding the oil spill, ie, The Minister of Mining Regulations No. 04 the year 1973 regarding Pollution prevention and handling waters in the exploration or oil and gas exploitation; The Minister of Transportation Regulations No. 58 the year 2013 regarding Response Pollution in waters and port; and Guideline Work System No. PTK-005/BP00000/2011 (Revision-01) regarding Oil Spill Response. Based on some guidelines and regulations mention above, some information included under the oil spill response plan are; operation area description, oil spill risk assessment, oil characteristic, oil spill modeling, Environmental Sensitivity Index (ESI), oil spill response strategy, oil spill response equipment, oil spill response organization, notification and activation of oil spill response organization, training and exercise, oil spill response termination.

Oil spill modeling is a key in determining the effective and efficient strategy to the oil spill response [6]. Model is one of the forms to simulate a symptom or structure or system, or abstraction of the real system. Another definition of the model is one of the forms that makes to imitate a symptom of the process. Oil spill modeling is generated on the fundamental equation of hydrodynamics:

$$
\partial \mathrm{s} / \partial \mathrm{t}+\partial \rho / \partial \mathrm{x}+\partial \mathrm{q} / \partial \mathrm{y}=\partial \mathrm{d} / \partial \mathrm{t}
$$

\footnotetext{
* Corresponding author: soesilo@indo.net.id
} 
First order show of water elevation, second order, and third order show the changes in density in $\mathrm{x}$ and $\mathrm{y}-$ direction, and fourth order shows the changes in water depth [7, 8]. But the fundamental equation of hydrodynamics, oil spill modeling also considering the weathering process of oil in waters [9]. Three main weathering condition of oil in waters are spreading, evaporation, and dispersion [9].

\section{Material Method}

Methods used are quantitative because the data will be analyzed using a statistic or model. Determination of location, Cilacap Regency waters have been carried out with purposive sampling method with criteria of the oil spill incident have been occured more than 2 times in past 10 years. Data for oil spill modeling parameter done by case review and oil spill risk assessment calculation. Oil spill modeling has done by oil spill modeling software, OILMAP. Data analysis was done by overlaying oil spill modeling results with the land use map of Cilacap Regency.

OILMAP is oil spill modeling system that use to predict oil movement and condition in waters [9]. These systems consist of an integrated component, Geographic Information System (GIS), environmental data system (EDS), spill scenario definitions, and spills models which show in Figure 1.

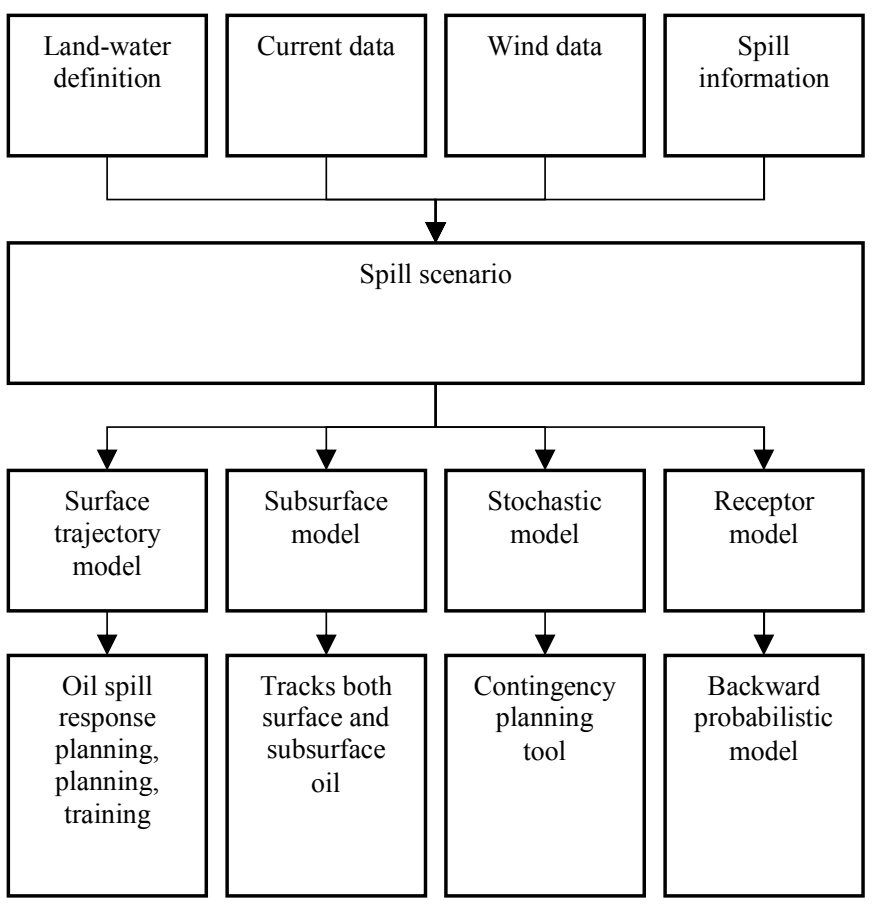

Fig. 1. Component Diagram of OILMAP

\section{Results and Discussion}

Mention above that the oil spill has occurred in Cilacap Regency for several time. The historical data will show in Table 1.
Table 1. Historical Data of Oil Spill Incident in Cilacap Regency

\begin{tabular}{|c|c|c|c|}
\hline No & Year & $\begin{array}{c}\text { Estimated } \\
\text { Spill Volume }\end{array}$ & Pollutant \\
\hline 1. & 2000 & 9000 tonnes & Asphalt \\
\hline 2. & 2004 & 8000 barrels & Crude oil \\
\hline 3. & 2007 & 30 barrels & Water ballast \\
\hline 4. & 2008 & 5 barrels & Crude oil \\
\hline 5. & 2010 & 10 barrels & Crude oil \\
\hline 6. & 2015 & Unknown & Crude oil \\
\hline 7. & 2016 & Unknown & Tarball \\
\hline
\end{tabular}

Oil spill modeling parameter that uses to predict oil spill movement in waters using OILMAP will show in Table 2.

Table 2. Oil Spill Modeling Parameter

\begin{tabular}{|c|c|}
\hline Parameter & Oil Spill Scenario \\
\hline Location & Cilacap Regency Waters \\
\hline $\begin{array}{c}\text { Waters } \\
\text { Temperature }\end{array}$ & $29^{\circ} \mathrm{C}$ \\
\hline Oil Type & Crude oil \\
\hline Oil Spill Volume & 8000 barrels \\
\hline Period Analysis & $\begin{array}{c}\text { Northwest (December-February) } \\
\text { First Transitional (March-April) } \\
\text { Southeast (May-September) } \\
\text { Second Transitional (October-November) }\end{array}$ \\
\hline
\end{tabular}

Oil spill movement generated by sea wind and sea current. The influence of wind speed on oil spill movement in waters about $3 \%$ and speed the current about $100 \%$ [2]. Statistic validation of the wind data done to compare the modeling data and reference data using Absolute Mean Error (AME) calculation:

$$
\mathrm{AME}=\frac{|\mathrm{S}-\mathrm{R}|}{\mathrm{R}} \times 100 \%
$$

AME as Absolute Mean Error, $\mathrm{S}$ as an average of simulation data, and $\mathrm{R}$ as an average of reference data. AME score for the validation is $2.21 \%$. 
Table 3. Validation of Simulation Data

\begin{tabular}{|c|c|c|c|c|c|c|c|c|c|c|c|}
\hline \multirow{4}{*}{ Date } & \multirow{4}{*}{ Time } & \multicolumn{2}{|c|}{ Reference Data } & \multicolumn{2}{|c|}{ Simulation Data } & \multirow[t]{2}{*}{ Date } & \multirow[t]{2}{*}{ Time } & \multirow{2}{*}{$\begin{array}{c}\text { Wind } \\
\text { Speed } \\
\text { (Knots) }\end{array}$} & \multirow{2}{*}{$\begin{array}{c}\text { Wind } \\
\text { Direction }\end{array}$} & \multirow{2}{*}{$\begin{array}{c}\text { Wind } \\
\text { Speed } \\
\text { (Knots) }\end{array}$} & \multirow{2}{*}{$\begin{array}{c}\text { Wind } \\
\text { Direction }\end{array}$} \\
\hline & & Wind & & Wind & & & & & & & \\
\hline & & Speed & $\begin{array}{l}\text { Wind } \\
\text { Dirstion }\end{array}$ & Speed & $\begin{array}{l}\text { Wind } \\
\text { Dirntion }\end{array}$ & $14 / 07$ & $6 \mathrm{AM}$ & 7 & $E$ & 11 & SE \\
\hline & & (Knots) & & (Knots) & & $14 / 07$ & $7 \mathrm{AM}$ & 7 & ESE & 11 & SE \\
\hline $11 / 07$ & $11 \mathrm{AM}$ & 6 & $\mathrm{E}$ & 7 & SE & $14 / 07$ & $8 \mathrm{AM}$ & 8 & ESE & 11 & SE \\
\hline $11 / 07$ & $12 \mathrm{AM}$ & 8 & $E$ & 6 & SE & $14 / 07$ & $9 \mathrm{AM}$ & 9 & ESE & 11 & SE \\
\hline $11 / 07$ & $1 \mathrm{PM}$ & 9 & $E$ & 6 & SE & $14 / 07$ & $10 \mathrm{AM}$ & 10 & ESE & 10 & $\mathrm{SE}$ \\
\hline $11 / 07$ & $2 \mathrm{PM}$ & 10 & ESE & 6 & SE & $14 / 07$ & $11 \mathrm{AM}$ & 10 & ESE & 9 & SE \\
\hline
\end{tabular}

\begin{tabular}{|c|c|c|c|c|c|}
\hline $11 / 07$ & $3 \mathrm{PM}$ & 10 & $\mathrm{ESE}$ & 5 & ESE \\
\hline $11 / 07$ & $4 \mathrm{PM}$ & 9 & $\mathrm{ESE}$ & 5 & $\mathrm{E}$ \\
\hline
\end{tabular}

\begin{tabular}{|c|c|c|c|c|c|}
\hline $11 / 07$ & $4 \mathrm{PM}$ & 9 & $\mathrm{ESE}$ & 5 & $\mathrm{E}$ \\
\hline $11 / 07$ & $5 \mathrm{PM}$ & 9 & $\mathrm{SE}$ & 4 & $\mathrm{E}$ \\
\hline $11 / 07$ & $6 \mathrm{PM}$ & 8 & $\mathrm{SE}$ & 4 & $\mathrm{E}$ \\
\hline $11 / 07$ & $7 \mathrm{PM}$ & 7 & $\mathrm{ESE}$ & 4 & $\mathrm{E}$ \\
\hline
\end{tabular}

\begin{tabular}{|c|c|c|c|c|c|}
\hline $11 / 07$ & 7 PM & 7 & ESE & 4 & E \\
\hline $11 / 07$ & 8 PM & 7 & ESE & 4 & E \\
\hline $11 / 07$ & 9 PM & 7 & ESE & 3 & E \\
\hline $11 / 07$ & $10 P M$ & 7 & & 3 & \\
\hline
\end{tabular}

\begin{tabular}{|c|c|c|c|c|c|}
\hline $11 / 07$ & $9 \mathrm{PM}$ & 7 & $\mathrm{ESE}$ & 3 & $\mathrm{E}$ \\
\hline $11 / 07$ & $10 \mathrm{PM}$ & 7 & $\mathrm{E}$ & 3 & $\mathrm{E}$ \\
\hline $11 / 07$ & $11 \mathrm{PM}$ & 6 & $\mathrm{E}$ & 3 & $\mathrm{E}$ \\
\hline $11 / 07$ & $12 \mathrm{PM}$ & 6 & $\mathrm{E}$ & 3 & $\mathrm{ENE}$ \\
\hline $12 / 07$ & $1 \mathrm{AM}$ & 6 & $\mathrm{ENE}$ & 3 & $\mathrm{E}$ \\
\hline $12 / 07$ & $2 \mathrm{AM}$ & 5 & $\mathrm{EE}$ & 5 & $\mathrm{SE}$ \\
\hline
\end{tabular}

\begin{tabular}{|c|c|c|c|c|c|}
\hline $12 / 07$ & 2 AM & 5 & ENE & 5 & SE \\
\hline $12 / 07$ & 3 AM & 5 & ENE & 6 & SE \\
\hline $12 / 07$ & 4 AM & 5 & ENE & 7 & SE \\
\hline
\end{tabular}

\begin{tabular}{|c|c|c|c|c|c|}
\hline $12 / 07$ & $4 \mathrm{AM}$ & 5 & $\mathrm{ENE}$ & 7 & $\mathrm{SE}$ \\
\hline $12 / 07$ & $5 \mathrm{AM}$ & 5 & $\mathrm{NE}$ & 8 & $\mathrm{SE}$ \\
\hline $12 / 07$ & $6 \mathrm{AM}$ & 5 & $\mathrm{NE}$ & 9 & $\mathrm{SE}$ \\
\hline $12 / 07$ & $7 \mathrm{AM}$ & 5 & $\mathrm{NE}$ & 9 & $\mathrm{SE}$ \\
\hline $12 / 07$ & $8 \mathrm{AM}$ & 5 & $\mathrm{ENE}$ & 10 & $\mathrm{SE}$ \\
\hline $12 / 07$ & $9 \mathrm{AM}$ & 6 & $\mathrm{E}$ & 10 & $\mathrm{SE}$ \\
\hline $12 / 07$ & $10 \mathrm{AM}$ & 7 & $\mathrm{ESE}$ & 9 & $\mathrm{SE}$ \\
\hline $12 / 07$ & $11 \mathrm{AM}$ & 7 & $\mathrm{ESE}$ & 9 & $\mathrm{SE}$ \\
\hline $12 / 07$ & $12 \mathrm{AM}$ & 8 & $\mathrm{SE}$ & 8 & $\mathrm{SE}$ \\
\hline $12 / 07$ & $1 \mathrm{PM}$ & 9 & $\mathrm{SE}$ & 8 & $\mathrm{SE}$ \\
\hline $12 / 07$ & $2 \mathrm{PM}$ & 9 & $\mathrm{SE}$ & 7 & $\mathrm{SE}$ \\
\hline $12 / 07$ & $3 \mathrm{PM}$ & 10 & $\mathrm{SE}$ & 7 & $\mathrm{SE}$ \\
\hline $12 / 07$ & $4 \mathrm{PM}$ & 10 & $\mathrm{SE}$ & 7 & $\mathrm{SE}$ \\
\hline $12 / 07$ & $5 \mathrm{PM}$ & 9 & $\mathrm{SE}$ & 7 & $\mathrm{SE}$ \\
\hline $12 / 07$ & $6 \mathrm{PM}$ & 8 & $\mathrm{SE}$ & 6 & $\mathrm{SE}$ \\
\hline $12 / 07$ & $7 \mathrm{PM}$ & 7 & $\mathrm{ESE}$ & 6 & $\mathrm{ESE}$ \\
\hline $12 / 07$ & $8 \mathrm{PM}$ & 7 & $\mathrm{ESE}$ & 6 & $\mathrm{ESE}$ \\
\hline $12 / 07$ & $9 \mathrm{PM}$ & 7 & $\mathrm{SE}$ & 6 & $\mathrm{SS}$ \\
\hline
\end{tabular}

\begin{tabular}{|c|c|c|c|c|c|}
\hline $12 / 07$ & $8 \mathrm{PM}$ & 7 & ESE & 6 & ESE \\
\hline $12 / 07$ & $9 \mathrm{PM}$ & 7 & $\mathrm{ESE}$ & 6 & ESE \\
\hline $12 / 07$ & $10 \mathrm{PM}$ & 7 & $\mathrm{E}$ & 7 & $\mathrm{E}$ \\
\hline
\end{tabular}

\begin{tabular}{|c|c|c|c|c|c|}
\hline $12 / 07$ & $10 \mathrm{PM}$ & 7 & $\mathrm{E}$ & 7 & $\mathrm{E}$ \\
\hline $12 / 07$ & $11 \mathrm{PM}$ & 7 & $\mathrm{E}$ & 7 & $\mathrm{E}$ \\
\hline $12 / 07$ & $12 \mathrm{PM}$ & 7 & $\mathrm{E}$ & 7 & $\mathrm{E}$ \\
\hline $13 / 07$ & $1 \mathrm{AM}$ & 7 & $\mathrm{E}$ & 9 & $\mathrm{E}$ \\
\hline $13 / 07$ & $2 \mathrm{AM}$ & 6 & $\mathrm{E}$ & 10 & $\mathrm{ESE}$ \\
\hline
\end{tabular}

\begin{tabular}{|c|c|c|c|c|c|}
\hline $13 / 07$ & $1 \mathrm{AM}$ & 7 & $\mathrm{E}$ & 9 & $\mathrm{E}$ \\
\hline $13 / 07$ & $2 \mathrm{AM}$ & 6 & $\mathrm{E}$ & 10 & $\mathrm{ESE}$ \\
\hline $13 / 07$ & $3 \mathrm{AM}$ & 6 & $\mathrm{E}$ & 11 & $\mathrm{ESE}$ \\
\hline
\end{tabular}

\begin{tabular}{|c|c|c|c|c|c|}
\hline $13 / 07$ & $3 \mathrm{AM}$ & 6 & $\mathrm{E}$ & 11 & $\mathrm{ESE}$ \\
\hline $13 / 07$ & $4 \mathrm{AM}$ & 6 & $\mathrm{E}$ & 11 & $\mathrm{SE}$ \\
\hline $13 / 07$ & $5 \mathrm{AM}$ & 7 & $\mathrm{E}$ & 12 & $\mathrm{SE}$ \\
\hline $13 / 07$ & $6 \mathrm{AM}$ & 7 & $\mathrm{E}$ & 12 & $\mathrm{SE}$ \\
\hline
\end{tabular}

\begin{tabular}{|c|c|c|c|c|c|}
\hline $13 / 07$ & $5 \mathrm{AM}$ & 7 & $\mathrm{E}$ & 12 & $\mathrm{SE}$ \\
\hline $13 / 07$ & $6 \mathrm{AM}$ & 7 & $\mathrm{E}$ & 12 & $\mathrm{SE}$ \\
\hline $13 / 07$ & $7 \mathrm{AM}$ & 7 & $\mathrm{E}$ & 12 & $\mathrm{SE}$ \\
\hline $13 / 07$ & $8 \mathrm{AM}$ & 8 & $\mathrm{E}$ & 12 & $\mathrm{SE}$ \\
\hline $13 / 07$ & $9 \mathrm{AM}$ & 9 & $\mathrm{E}$ & 12 & $\mathrm{SE}$ \\
\hline
\end{tabular}

\begin{tabular}{|c|c|c|c|c|c|}
\hline $13 / 07$ & 8 AM & 8 & E & 12 & SE \\
\hline $13 / 07$ & 9 AM & 9 & E & 12 & SE \\
\hline $13 / 07$ & 10 AM & 10 & ESE & 11 & SE \\
\hline $13 / 07$ & 11 AM & 11 & ESE & 10 & SE \\
\hline $13 / 07$ & 12 AM & 11 & ESE & 9 & SE \\
\hline $13 / 07$ & 1 PM & 12 & SE & 9 & SE \\
\hline $13 / 07$ & 2 PM & 12 & SE & 9 & SE \\
\hline $13 / 07$ & 3 PM & 12 & SE & 9 & SE \\
\hline $13 / 07$ & 4 PM & 11 & SE & 9 & SE \\
\hline $13 / 07$ & 5 PM & 11 & SE & 9 & SE \\
\hline $13 / 07$ & 6 PM & 9 & SE & 9 & SE \\
\hline $13 / 07$ & 7 PM & 8 & ESE & 9 & SE \\
\hline $13 / 07$ & 8 PM & 8 & ESE & 9 & SE \\
\hline $13 / 07$ & 9 PM & 8 & ESE & 8 & SE \\
\hline $13 / 07$ & 10 PM & 8 & ESE & 8 & ESE \\
\hline $13 / 07$ & $11 \mathrm{PM}$ & 8 & ESE & 9 & ESE \\
\hline $13 / 07$ & 12 PM & 8 & ESE & 9 & E \\
\hline $14 / 07$ & 1 AM & 8 & E & 9 & SE \\
\hline $14 / 07$ & $2 \mathrm{AM}$ & 8 & E & 10 & SE \\
\hline $14 / 07$ & $3 \mathrm{AM}$ & 8 & $\mathrm{E}$ & 10 & SE \\
\hline $14 / 07$ & $4 \mathrm{AM}$ & 7 & $\mathrm{E}$ & 10 & SE \\
\hline $14 / 07$ & $5 \mathrm{AM}$ & 7 & $\mathrm{E}$ & 11 & SE \\
\hline
\end{tabular}

Simulation results of the oil spill model for each monsoon are shown in Figure 2-3. Figure 2 shows the oil spill model results in Northwest monsoon. In Northwest monsoon, the oil spill in waters will move toward east and southeast, Indian Ocean. Based on Cilacap Regency land use, sub-district that potentially impacted by the oil spill are Cilacap Selatan, Cilacap Tengah, Kecamatan Cilacap Utara, Adipala, Binangun, and Nusawungu. Area that potentially impacted utilized as tourism beach, forest, and settlement.

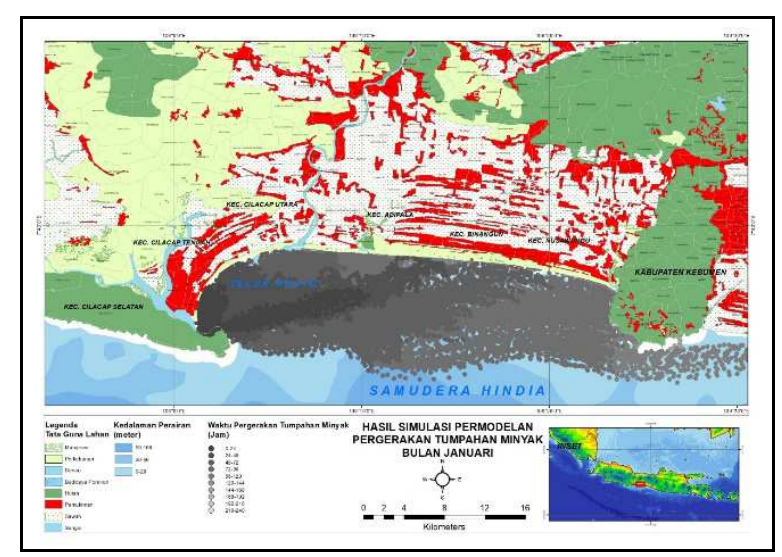

Fig. 2. Oil Spill Model in Northwest Monsoon

Figure 3 shows the oil spill model results in Southeast monsoon, the oil spill in waters will move toward west and northwest, Segara Anakan Lagoon. Based on Cilacap Regency land use, sub-district that potentially impacted by oil spill are Cilacap Selatan and Kecamatan Cilacap Tengah. Area that potentially impacted utilized as tourism beach, forest, and settlement.

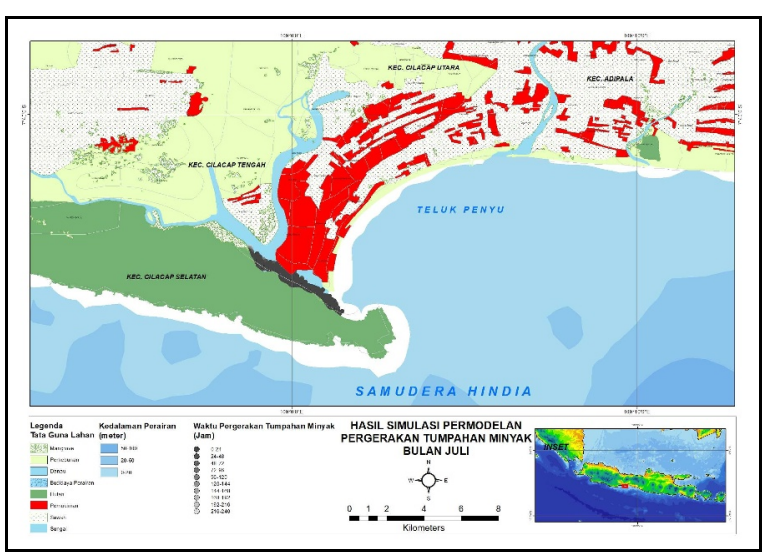

Fig. 3. Oil Spill Model in Southeast Monsoon 
Another result of OILMAP software is weathering process information. Figure 4 shows the oil weathering results in single trajectory modeling. After 240 hours released to waters, the crude oil will evaporate about $19,2 \%$, stranded at shoreline about $79,76 \%$.

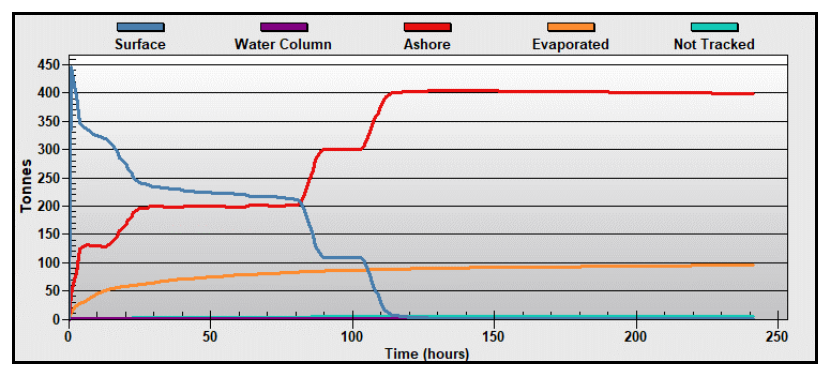

Fig. 4. Weathering of Crude Oil

The viscosity of the oil is a measure of its resistance to internal flow and an indication of its oiliness in the lubrication of surface. Figure 5 shows the viscosity of crude oil, by the time the viscosity of crude oil will increase. It means that once the crude oil spilled to waters, the oil gets thicker and the flow will be decreased. This will affect the emulsification rate, where the volume of oil mixed with water will increase. Related to the preparation of oil spill response equipment, this will affect the amount of oil skimmer and oil temporary storage tank.

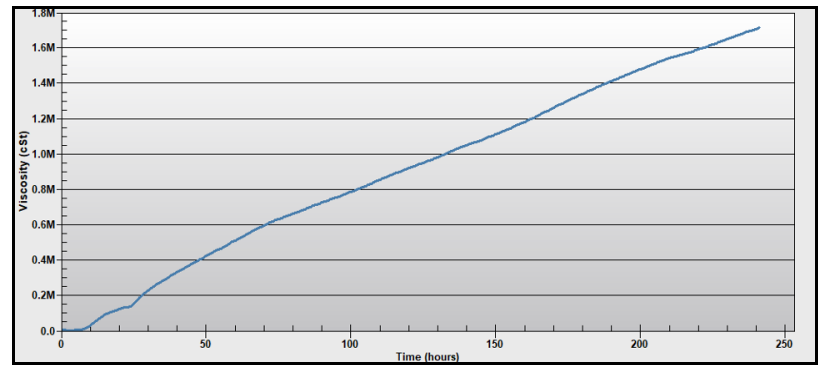

Fig. 5. The Viscosity of Crude Oil

\section{Conclusion}

The results showed that in Northwest monsoon, the oil spill will move toward the Indian Ocean and in Southeast monsoon, the oil spill will move toward Segara Anakan Lagoon. The response strategy has to develop to protect the tourism area of Teluk Penyu beach and fishing ground around Segara Anakan Lagoon based on oil spill modeling.
This research is funded by the Grant of Indexed International Publication for Final Project of Students/Publikasi Terindeks Internasional Untuk Tugas Akhir Mahasiswa (PITTA) Universitas Indonesia 2018 with contract number 2585/UN2.R3.1/HKP.05.00/2018

\section{References}

1. Peraturan Presiden Republik Indonesia No 109 Tahun 2006 tentang Penanggulangan Keadaan Darurat Tumpahan Minyak di Laut (2006).

2. Hyder, K., S. Wright., M. Kirby., J, Brant. The Role of Citizen Science in Monitoring Small Scale Pollution Event. Marine Pollution Buletin.120. 5157 (2017).

3. Ishvina, I. B., M. S. Kuyukina, A. V. Krivoruchko, A. A. Elkin, S. O. Makarov, C. J. Cunningham, T. A. Peshkur, R. M. Atlas, J. C. Philip. Oil spill problems and sustainable response strategies through new technologies. Environ. Sci.: Processes Impacts. 17. 1201-1219. (2015).

4. Negro, C. Garcia., S. Villasante, A. Panela, Carballo, G. Rodriguez. Estimating the Economic Impact of The Prestige Oil Spill on The Death Coast Spain Fisheries. El Sevier (2008).

5. Kip, S.H. Oil Spill Contingency Plan. 7th Offshore South East Asia Conference (1988).

6. White, I., Molloy, Fionn. 2002. Ships and The Marine Environment. ITOPF. (2002).

7. IPIECA. Impact of Oil Spills on Marine Ecology. IPIECA (2016).

8. RPS. OILMAP Training Modul. RPS APASA PTY LTD. Australia (2014).

9. RPS. OILMAP Training Modul. RPS APASA PTY LTD. Australia (2014).

\footnotetext{
*Corresponding author: soesilo@,indo.net.id
} 